\title{
Fenologia reprodutiva de Carapichea ipecauanha (Brot.) L. Andersson e sua correlação com a temperatura média do ar e precipitação pluviométrica
}

\author{
Reproductive phenology of Carapichea ipecauanha (Brot.) L. Andersson and its correlation with \\ average air temperature and rainfall \\ Fenología reproductiva de Carapichea ipecauanha (Brot.) L. Andersson y su correlación con la \\ temperatura media del aire y las precipitaciones
}

\author{
Meiciane Ferreira Campelo \\ ORCID: https://orcid.org/0000-0002-9566-3741 \\ Universidade Federal do Pará, Brasil \\ E-mail: meicianecampelo@gmail.com \\ Osmar Alves Lameira \\ ORCID: https://orcid.org/0000-0001-8370-8562 \\ Empresa Brasileira de Pesquisa Agropecuária Amazônia Oriental, Brasil \\ E-mail: osmar.lameira @embrapa.br \\ Ruanny Karen Vidal Pantoja Portal Moreira \\ ORCID: https://orcid.org/0000-0001-7511-4377 \\ Universidade Federal do Pará, Brasil \\ E-mail: ruanny_vidal@hotmail.com \\ Allan Cristiam Santos Ramires \\ ORCID: https://orcid.org/0000-0002-0953-7221 \\ Universidade Federal Rural da Amazonia, Brasil \\ E-mail: allanramires15@gmail.com
}

\begin{abstract}
Resumo
A espécie Carapichea ipecacuanha é uma referência entre as plantas medicinais com princípios ativos de relevância econômica devido à presença de dois alcaloides isoquinolínicos: a emetina e a cefalina. Sendo a fenologia o ramo da ecologia considerada fundamental para o entendimento da dinâmica das comunidades vegetais e estratégias para o uso sustentável de espécies. O objetivo deste trabalho foi registrar as fenofases de Carapichea ipecacuanha, correlacionando-as com os elementos climáticos, com a finalidade de indicar a época mais adequada para a coleta $\mathrm{e}$ utilização do material vegetal da espécie. As frequências foram registradas semanalmente no período de 2015 a 2019 , avalivou-se o índice de atividade, correlação entre fenofases e elementos climáticos e percentuais de dias de fenofases. Os resultados evidenciam que a espécie, flora e frutifica todos os meses do ano, a atividade fenológica exibiu diferenças entre acessos, o índice de correlação de Spearman apontou correlação significativa entre frutificação e fatores climáticos. Registrou-se variação no padrão de ocorrência de número de dias de floração e frutificação entre os grupos de acessos, sendo o número de dias máximo de ocorrência de floração notada em outubro, e frutificação com destaque em março e abril. Contudo sugere-se a coleta de material vegetal da espécie para uso medicinal nos meses de agosto e setembro, cujo registro de número de dias que ocorrem as fenofases sofre reduções. È pertinente executar posteriores estudos de correlação da fenologia com eventos climáticos considerando individualmente o desempenho de cada acesso.
\end{abstract}

Palavras-chave: Ipeca; Fenologia reprodutiva; Pluviosidade; Temperatura.

\begin{abstract}
The species Carapichea ipecacuanha is a reference among medicinal plants with active principles of economic relevance due to the presence of two isoquinoline alkaloids: emetin and cephaline. Phenology being the branch of ecology considered fundamental for understanding the dynamics of plant communities and strategies for the sustainable use of species. The objective of this work was to register the phenophases of Carapichea ipecacuanha, correlating them with the climatic elements, in order to indicate the most suitable time for the collection and use of the plant material of the species. The frequencies were recorded weekly in the period from 2015 to 2019, the activity index, correlation between phenophases and climatic elements and percentages of days of phenophases were evaluated. The results show that the species, flora and fruit every month of the year, the phenological activity showed differences between accessions, the Spearman correlation index showed a significant correlation between fruiting and climatic factors. Variation in the pattern of occurrence of the number of days of flowering and fruiting was recorded between the accession groups, with the maximum number of days of flowering occurring in October and November, and fruiting with a peak especially in March and April. However, it is suggested the collection of plant material of the
\end{abstract}


species for medicinal in the months of August and September, whose registration of the number of days that occur the phenophases undergoes reductions. It is pertinent to carry out further studies on the correlation of phenology with climatic events considering the each access performance individually.

Keywords: Ipeca; Reproductive phenology; Rainfall; Temperature.

\section{Resumen}

La especie Carapichea ipecacuanha es una referencia entre las plantas medicinales con principios activos de relevancia económica debido a la presencia de dos alcaloides isoquinolínicos: emetina y cefalina. La fenología es la rama de la ecología considerada fundamental para comprender la dinámica de las comunidades vegetales y las estrategias para el uso sostenible de las especies. El objetivo de este trabajo fue registrar las fenofases de Carapichea ipecacuanha, correlacionándolas con los elementos climáticos, con el fin de indicar el momento más adecuado para la recolección y uso del material vegetal de la especie. Las frecuencias se registraron semanalmente en el período de 2015 a 2019, se evaluó el índice de actividad, correlación entre fenofases y elementos climáticos y porcentajes de días de fenofases. Los resultados muestran que la especie, flora y fruto todos los meses del año, la actividad fenológica mostró diferencias entre accesiones, el índice de correlación de Spearman mostró una correlación significativa entre factores fructíferos y climáticos. Se registró variación en el patrón de ocurrencia del número de días de floración y fructificación entre los grupos de accesiones, registrándose el número máximo de días de floración en octubre y noviembre, y la fructificación con un destaque en marzo y abril. Sin embargo, se sugiere la recolección de material vegetal de la especie para uso medicinal en los meses de agosto y septiembre, cuyo registro del número de días que ocurren las fenofases sufre reducciones. Es pertinente realizar más estudios sobre la correlación de la fenología con los eventos climáticos considerando el desempeño del cada acceso de manera individual.

Palabras clave: Ipeca; Fenología reproductiva; Lluvia; La temperatura.

\section{Introdução}

A espécie Carapichea ipecacuanha conhecida popularmente como ipeca, da familia Rubiaceae, é uma referência entre as plantas medicinais com princípios ativos de relevância econômica (Ferreira et al., 2012). A ipeca é uma planta que possui atividade farmacológica devido à presença de dois alcaloídes isoquinilínicos: a emetina e a cefalina, seu uso principal é como emético, essa propriedade deve-se mais à cefalina que à emetina, provocando vômito em ocasiões de intoxicação, com o objetivo de impossibilitar ou diminuir a absorção do agente tóxico ingerido e que ainda está no estômago (Silva, 2014).

Desta forma, possui um grande potencial econômico, característica garantida pela comercialização de suas raízes (Ricardo et al., 2017), sendo a parte que possui maior concentração desses alcaloídes. E de acordo com Rocha \& Lameira (2011) ao arrancar a raiz, a espécie não se regenera e diante disso a ipeca é uma espécie ameaçada de erosão genética ou em vias de extinção, pelo fato de ter sofrido intenso processo extrativo por mais de dois séculos, por meio do desmatamento da Mata Atlântica e a abertura de novas fronteiras agrícolas diminuindo suas áreas de ocorrência natural; a forma extrativista com plantios de ipeca inexpressivos, sem manejo adequado; e a dificuldade do cultivo convencional da espécie, frente à grande demanda de mercado. (Rocha \& Lameira, 2011).

Com isso, a Fenologia é o ramo da ecologia considerada fundamental para o entendimento da dinâmica das comunidades vegetais, pois facilita o entendimento dos processos de regeneração e estratégias para o uso sustentável de espécies e avaliação de impactos climáticos (Tannus et al., 2006). Os estudos fenológicos abordam os diferentes eventos biológicos, dentre estes eventos estão o florescimento e a frutificação (Neves et al., 2010). A compreensão dos padrões fenológicos, comportamento anual e as relações das fenofases com as variações ambientais, fornecem informações para um manejo adequado e preservação de recursos genéticos (Vieira \& Carvalho, 2009).

Portanto, um dos objetivos da fenologia é estudar as relações entre os fenômenos biológicos periódicos e as condições meteorológicas ou climatológicas, analisando e comparando as variações temporais e geográficas, que determinam o aparecimento dos seres vivos como respostas às variações ambientais no tempo e no espaço (Pascale; Damário, 2004). Daí ser considerada uma ciência multidisciplinar (Morellato et al. 2016).

Os fatores climatológicos como a radiação solar, temperatura, pluviosidade e umidade relativa do ar são fatores limitantes no desenvolvimento fenológico de uma determinada espécie, podendo variar em diferentes períodos dentro de um 
mesmo ano (Forsthofer, 2004). Dentre os fatores climáticos aos quais as plantas são sensíveis destaca-se a temperatura do ar e precipitação que como fatores abióticos influenciam no crescimento (Zanon \& Finger, 2010).

Deste modo, o objetivo deste trabalho foi registrar as fenofases reprodutivas de Carapichea ipecacuanha pertencente ao Banco Ativo Germoplasma da Embrapa Amazônia Oriental, correlacioando com os elementos climáticos como a precipitação pluviométrica e temperatura média do ar, com a finalidade de indicar a época mais adequada para a coleta e utilização do material vegetal da espécie.

\section{Metodologia}

O estudo foi conduzido no Banco Ativo de Germoplasma (BAG) pertencente ao Horto de plantas medicinais da Embrapa Amazônia Oriental, situada no município de Belém, PA. O clima da apresenta temperatura média anual de $25,9^{\circ} \mathrm{C}$, variando entre $21{ }^{\circ} \mathrm{C}$ e $31,6{ }^{\circ} \mathrm{C}$, com umidade relativa do ar de $84 \%$ e precipitação pluviométrica anual de $2.900 \mathrm{~mm}$, com trimestre mais chuvoso entre os meses de janeiro e março (Peel et al., 2007).

O nome científico da espécie em estudo Carapichea ipecauanha (Brot.) L. foi atualizado nas bases de dados da Lista de Espécies da Flora do Brasil (Forzza, 2014) e do Mobot Tropicos, do Missouri Botanical Garden (Tropicos, 2014).A identificação botânica foi realizada no Laboratório de Botânica da Embrapa Amazônia Oriental, e as exsicatas, encontram-se no Herbário IAN da mesma instituição. O material de estudo apresenta cinco diferentes procedências de origem, Estados de Rondônia, Minas Gerais, Bahia, Rio de Janeiro e Mato Grosso (Alelo Vegetal, 2021), contudo no local de estudo todos foram submetidos às mesmas condiçoes de cultivo.

Avaliou-se a fenologia reprodutiva da ipeca através de registros realizados cinco vezes por semana ao longo do período de janeiro de 2015 a dezembro de 2019, desta forma obtendo os dados de comportamentos fenológicos de floração e frutificação, sendo o período de floração caracterizado desde a formação de botões florais até o final do período de antese das flores, e o período de frutificação determinado através da formação visível dos frutos até a sua queda. Os dados meteorológicos de precipitação pluviométrica e temperatura média do ar foram obtidos por meio do banco de dados da estação meteorológica da Embrapa Amazônia Oriental, onde foram utilizadas as médias mensais acumuladas (Figura 1). 
Figura1. Clima do Local de estudo, temperatura média do ar e precipitação pluviométrica média mensal e anual referente ao período de 2015 a2019, no município de Belém-PA.

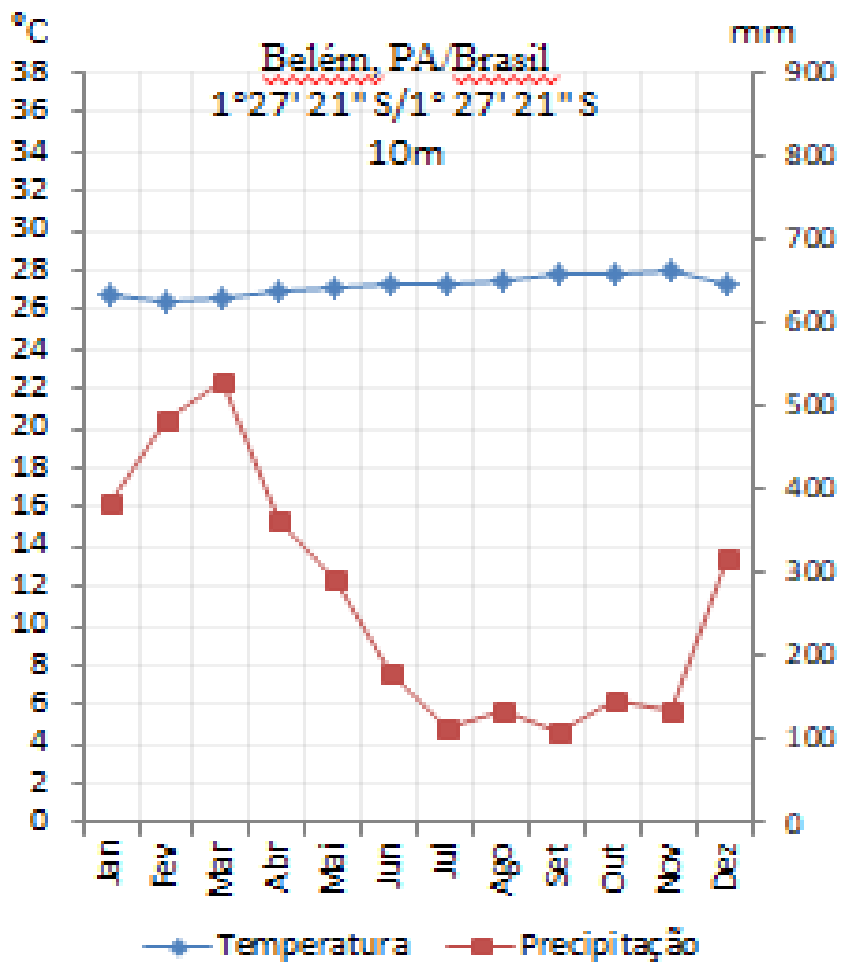

\begin{tabular}{c|c|c}
\multicolumn{3}{c}{ DADOS CLDMATOLÓGICOS } \\
2015-2019 \\
Meses & Precip (mm) & TMedia ( $\left.{ }^{\circ} \mathrm{C}\right)$ \\
\hline Jan & 384,43 & 26,8 \\
Fev & 484,50 & 26,4 \\
Mar & 531,43 & 26,6 \\
Abr & 364,15 & 26,9 \\
Mai & 294,58 & 27,2 \\
Jun & 181,93 & 27,3 \\
Jul & 115,03 & 27,3 \\
Ago & 136,78 & 27,4 \\
Set & 109,65 & 27,8 \\
Out & 149,23 & 27,9 \\
Nov & 134,50 & 28,0 \\
Dez & 318,40 & 27,3 \\
\hline Temperatura Média anual $-27,24$ \\
Precipitaçảo Média anual $-267,05$
\end{tabular}

Fonte: Estação Climatológica da Embrapa Amazônia Oriental.

Para a demonstração dos resultados foi seguido o índice de atividade descrito por Bencke \& Morellato, (2002), que consiste em notar a ocorrência ou a ausência do evento reprodutivo de uma população (neste trabalho referente á cada acesso). E o uso do termo "pico", ao longo do texto, refere-se ao pico de atividade, como o mês que apresenta o maior número de acessos em uma determinada fenofase.

Para averiguar a existência ou não de correlação significativa entre as fenofases e as variáveis abióticas foi utilizada a correlação de Spearman com o auxilio do software estatístico R (2020), onde o coeficiente de correlação (rho), com sinal negativo indica que as variáveis se correlacionam de maneira inversa, ou seja, o aumento de uma está correlacionado a decréscimo da outra, e a intensidade é classificada da seguinte maneira, os valores de rho entre 0,34 e 0,66 indicam relações fracas (rho fraca) e a partir de 0,67 relações fortes (rho forte) como sugerido por Cann (2002).E a elaboração de gráficos e tabelas com o auxilio do Microsoft Excel.

\section{Resultados e Discussão}

Os resultados dos elementos climáticos evidenciaram que a plusiovidade variou de 109,65 mm em setembro á 531,43 $\mathrm{mm}$ em março, e a temperatura média variou de $26,4^{\circ} \mathrm{C}$ em fevereiro á $28^{\circ} \mathrm{C}$ em novembro, sendo o índice de precipitação média e da temperatura média anual, registrados de $267,05 \mathrm{~mm}$ e $27,24^{\circ} \mathrm{C}$ (Figura 1).

A espécie Carapichea ipecacuanha, apresentou ocorrência das fenofases de reprodução todo o ano, porém com variações nos padrões fenológicos de floração e frutificação ao decorrer dos meses no período estudado (Figura 2, Tabela 1). Fortunato e Quirino (2016) obervaram a formação de flores e frutos durante o ano todo em Rubiaceas nos fragmentos de Mata Atlântica.

O maior percentual de acessos florando ocorreu de setembro á novembro, atingindo o pico em setembro $(91,52 \%)$. Os meses que apresentaram os mais expressivos percentuais de acessos frutificando foram de janeiro á março, sendo o pico 
registrado em fevereiro com 94,91\% de acessos com frutos (Figura 2). Resultados distintos aos encontrados neste trabalho foram descritos por Andrade (2020) no qual o padrão se mostrou contínuo na produção de flores e frutos apresentado pela comunidade da família Rubiaceae.

Figura 2. Padrão fenológico dos eventos reprodutivos de floração e frutificação da Ipeca.

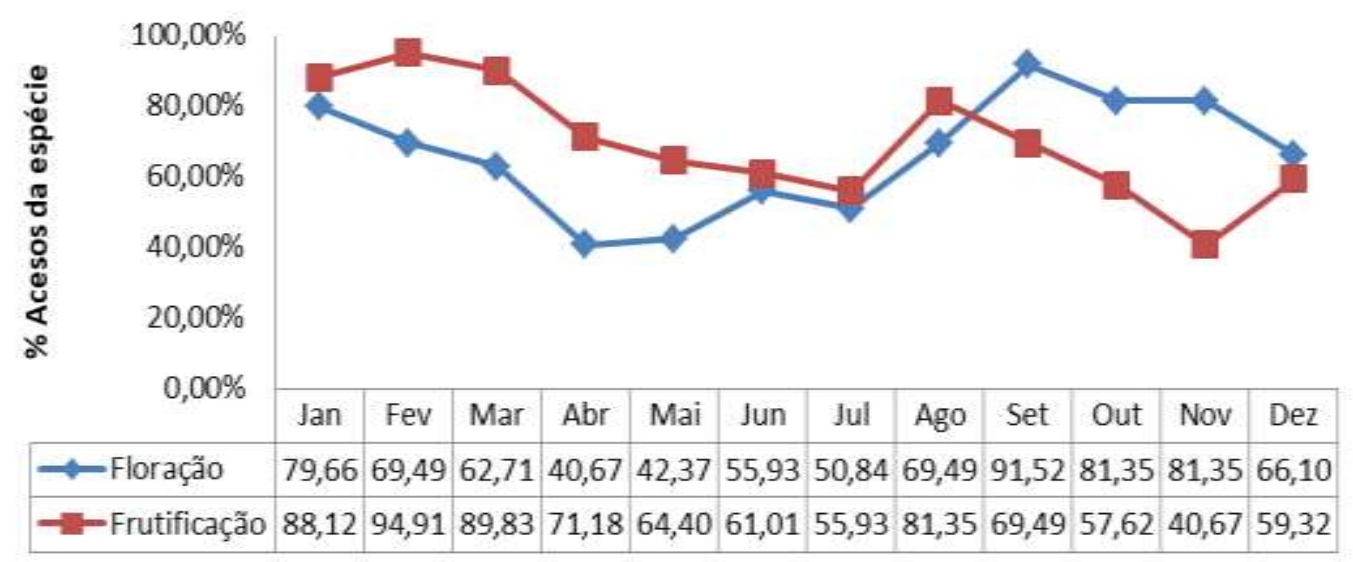

Fonte: Autores.

O índice de atividade representado na Tabela 1 expõe o registro de ocorrência ou não do evento reprodutivo em 66 acessos cultivados na mesma condicões no BAG, entretanto com origem em cinco Estados. Os resultados obitidos revelam diferenças na atividade fenológica dos eventos reprodutivos entre acessos, temos como exemplo 63 acessos que apresentaram variações no registro dos eventos de frutificação e floração, diferentemente dos acessos 811,812 e 814 que foram os únicos que apresentaram máxima constância das fenofases durante todos os meses do período avaliado. Assincronia entre acessos de Psychotria nuda (Rubiaceae) em resposta a frequência de todos os eventos fenológicos, exceto para folhas senescentes, foram identificados em estudos realizados por Ribeiro et al.,(2018).

Tabela 1. Atividade fenológica reprodutiva de acessos de Ipeca provenientes de cinco Estados, Brasil. $\left(\mathrm{N}^{\circ}=\right.$ número de indivíduos avaliados; floração = ०; frutificação $=*$ ).

\begin{tabular}{|c|c|c|c|c|c|c|c|c|c|c|c|c|c|}
\hline \multirow[t]{2}{*}{ Municípios } & \multirow[t]{2}{*}{ Acessos } & \multicolumn{12}{|c|}{ Meses } \\
\hline & & Jan & Fev & Mar & Abr & Mai & Jun & Jul & Ago & Set & Out & Nov & Dez \\
\hline \multirow[t]{11}{*}{$\mathrm{RO}$} & 819 & $\circ *$ & $*$ & $*$ & $\circ *$ & $\circ *$ & $*$ & * & $\circ *$ & $\circ *$ & $\circ *$ & $\circ$ & $\circ *$ \\
\hline & 845 & $\circ *$ & * & $\circ *$ & & & & & $\circ *$ & $\circ *$ & $\circ *$ & $\circ$ & \\
\hline & 610 & $*$ & * & $\circ *$ & * & & & & $*$ & $\circ *$ & & $*$ & $*$ \\
\hline & 612 & $\circ *$ & $\circ *$ & $*$ & * & $*$ & & & 。 & 。 & & 。 & \\
\hline & 826 & $\circ *$ & $\circ *$ & $\circ *$ & * & $\circ *$ & $*$ & $\circ *$ & $\circ *$ & $\circ *$ & $\circ$ & 。 & $\circ$ \\
\hline & 827 & $\circ *$ & $\circ *$ & $\circ *$ & $*$ & $\circ *$ & $*$ & $*$ & $\circ *$ & $\circ *$ & $\circ *$ & $\circ$ & $\circ *$ \\
\hline & 833 & $\circ *$ & $\circ *$ & $\circ *$ & $\circ *$ & $*$ & $\circ *$ & $\circ *$ & $\circ *$ & $\circ *$ & $\circ *$ & $\circ *$ & $\circ *$ \\
\hline & 834 & $\circ *$ & $\circ *$ & $\circ *$ & $*$ & $\circ *$ & $\circ *$ & $\circ *$ & $*$ & $\circ *$ & $\circ *$ & $\circ *$ & $\circ *$ \\
\hline & 836 & $\circ *$ & $\circ *$ & $\circ *$ & * & $*$ & $\circ *$ & $\circ *$ & $\circ *$ & $\circ *$ & $\circ *$ & $\circ$ & $\circ$ \\
\hline & 837 & $\circ *$ & $\circ *$ & $\circ *$ & $*$ & $*$ & $*$ & $*$ & $\circ *$ & $\circ *$ & & & \\
\hline & 838 & $\circ *$ & $*$ & $\circ *$ & $*$ & $*$ & $*$ & & $*$ & $\circ *$ & & $\circ$ & \\
\hline
\end{tabular}


Research, Society and Development, v. 10, n. 5, e7610514625, 2021

(CC BY 4.0) | ISSN 2525-3409 | DOI: http://dx.doi.org/10.33448/rsd-v10i5.14625

\begin{tabular}{|c|c|c|c|c|c|c|c|c|c|c|c|c|c|}
\hline & 839 & $\circ *$ & $\circ *$ & $\circ *$ & $\circ *$ & $\circ *$ & ०* & * & $\circ *$ & $*$ & & & \\
\hline & 775 & $\circ *$ & $\circ *$ & $\circ *$ & $*$ & $\circ *$ & $\circ *$ & $\circ *$ & $\circ *$ & $\circ$ & $\circ$ & $\circ$ & $\circ *$ \\
\hline & 776 & $*$ & $\circ *$ & $\circ *$ & $\circ *$ & $\circ *$ & $\circ *$ & $\circ *$ & $\circ$ & $\circ *$ & $\circ *$ & $\circ *$ & $\circ *$ \\
\hline & 777 & $\circ *$ & $*$ & $\circ *$ & $*$ & $*$ & $\circ$ & $\circ$ & $*$ & $\circ$ & $\circ$ & $\circ$ & $\circ$ \\
\hline & 801 & $\circ *$ & $*$ & $\circ *$ & & $\circ$ & ○ & $*$ & $\circ$ & $\circ *$ & $\circ$ & $\circ$ & $\circ *$ \\
\hline & 802 & $\circ *$ & $\circ *$ & $\circ *$ & $\circ *$ & o & ०* & $\circ *$ & ०* & $\circ *$ & $\circ *$ & $\circ *$ & $\circ *$ \\
\hline & 803 & $*$ & $\circ *$ & $\circ *$ & $*$ & $*$ & $*$ & $*$ & $*$ & 0 & $\circ *$ & $*$ & $\circ *$ \\
\hline & 806 & $\circ *$ & $\circ *$ & $\circ *$ & $*$ & $\circ *$ & * & $\circ *$ & $\circ *$ & $\circ$ & $\circ$ & $\circ$ & $\circ$ \\
\hline & 807 & $\circ *$ & $\circ *$ & $*$ & $*$ & $*$ & $\circ *$ & $\circ *$ & $\circ *$ & $\circ *$ & $\circ *$ & $\circ *$ & $\circ *$ \\
\hline & 808 & $\circ *$ & $\circ *$ & $\circ *$ & $\circ *$ & $\circ *$ & ०* & $\circ *$ & $\circ *$ & $\circ *$ & $\circ$ & $\circ$ & \\
\hline & 810 & $\circ *$ & $\circ *$ & $*$ & $*$ & $*$ & * & $*$ & & $\circ *$ & $\circ *$ & $\circ *$ & $\circ *$ \\
\hline & 811 & $\circ *$ & $\circ *$ & $\circ *$ & $\circ *$ & $\circ *$ & $\circ *$ & $\circ *$ & $\circ *$ & $\circ *$ & $\circ *$ & $\circ *$ & $\circ *$ \\
\hline & 812 & $\circ *$ & $\circ *$ & $\circ *$ & $\circ *$ & $\circ *$ & ०* & $\circ *$ & $\circ *$ & $\circ *$ & $\circ *$ & $\circ *$ & $\circ *$ \\
\hline & 814 & $\circ *$ & $\circ *$ & $\circ *$ & $\circ *$ & $\circ *$ & ०* & $\circ *$ & $\circ *$ & $\circ *$ & $\circ *$ & $\circ *$ & $\circ *$ \\
\hline & 815 & $\circ *$ & & $\circ *$ & $\circ *$ & $\circ *$ & $\circ$ & & $*$ & $\circ$ & $\circ *$ & $\circ$ & $\circ$ \\
\hline $\mathrm{MG}$ & 753 & $\circ *$ & $\circ *$ & $\circ *$ & $\circ *$ & $*$ & ०* & $\circ *$ & & 0 & $\circ$ & $\circ *$ & $\circ *$ \\
\hline B & 767 & $\circ *$ & $\circ *$ & $\circ *$ & $\circ *$ & $*$ & ○* & $*$ & ०* & $\circ *$ & $\circ *$ & $\circ$ & $*$ \\
\hline RJ & 745 & $\circ *$ & $\circ *$ & $\circ *$ & $\circ *$ & $*$ & o* & $\circ *$ & $\circ *$ & $\circ *$ & $\circ$ & $\circ *$ & ०* \\
\hline & 746 & $\circ *$ & $\circ *$ & $\circ *$ & $\circ *$ & $\circ *$ & ०* & $*$ & o* & $\circ *$ & $\circ *$ & $\circ *$ & $*$ \\
\hline & 751 & $\circ *$ & $\circ *$ & $\circ *$ & $\circ *$ & & & . & $\circ$ & $\circ$ & $\circ$ & $\circ$ & $\circ *$ \\
\hline MT & 571 & $\circ *$ & $*$ & o* & & & $\circ$ & $\circ$ & $*$ & $\circ *$ & $\circ *$ & $\circ$ & \\
\hline & 572 & $\circ$ & $\circ *$ & $*$ & & & & & $\circ *$ & $*$ & $\circ$ & $\circ$ & $\circ *$ \\
\hline & 573 & $\circ$ & $\circ *$ & $\circ *$ & & & & $\circ$ & $\circ$ & $\circ$ & $*$ & & $\circ *$ \\
\hline & 574 & $\circ$ & $*$ & $*$ & & & & & $\circ *$ & $\circ$ & & $\circ$ & $\circ *$ \\
\hline & 575 & $\circ *$ & $\circ *$ & $\circ$ & $\circ$ & $\circ$ & ○ & & $\circ *$ & $\circ$ & $\circ *$ & $\circ$ & $\circ$ \\
\hline & 576 & & $*$ & & & & & & $\circ *$ & $\circ *$ & $\circ *$ & $\circ *$ & $\circ *$ \\
\hline & 577 & $\circ *$ & $\circ *$ & $\circ *$ & $*$ & $*$ & ०* & & $*$ & $\circ *$ & & $\circ$ & $\circ *$ \\
\hline & 580 & & $*$ & $*$ & & & & & $\circ *$ & $\circ *$ & $\circ *$ & & \\
\hline & 689 & $*$ & & $*$ & $\circ *$ & $*$ & & & $*$ & $\circ *$ & & $\circ$ & \\
\hline & 690 & $\circ *$ & $\circ *$ & $\circ$ & $\circ *$ & $\circ *$ & $\circ *$ & $\circ$ & $\circ *$ & $\circ$ & $\circ *$ & $\circ *$ & $\circ *$ \\
\hline & 693 & $\circ *$ & $\circ *$ & $\circ *$ & $\circ$ & & & & $\circ$ & $\circ$ & $\circ *$ & $\circ *$ & $\circ *$ \\
\hline & 694 & $\circ *$ & * & $\circ *$ & & & & & $*$ & $\circ$ & $\circ$ & $\circ$ & \\
\hline & 695 & $*$ & $*$ & & & $\circ$ & $\circ$ & & $*$ & $\circ *$ & $\circ$ & $\circ *$ & $*$ \\
\hline & 696 & $\circ *$ & $\circ *$ & $\circ *$ & * & $\circ *$ & $*$ & ० & $\circ$ & $\circ *$ & $\circ *$ & $\circ *$ & $\circ *$ \\
\hline & 594 & $*$ & $\circ$ & & & & & & $\circ *$ & $\circ *$ & $\circ *$ & $\circ$ & $\circ$ \\
\hline
\end{tabular}




\begin{tabular}{|c|c|c|c|c|c|c|c|c|c|c|c|c|}
\hline 589 & o* & $\circ *$ & * & $\circ *$ & & $\circ *$ & $\circ$ & & $*$ & $\circ *$ & $\circ$ & o \\
\hline 590 & $\circ *$ & $\circ *$ & $\circ *$ & $*$ & $\circ *$ & $\circ *$ & $\circ *$ & o* & o & $\circ *$ & $\circ *$ & \\
\hline 707 & $\circ *$ & $\circ *$ & * & $*$ & $\circ *$ & $\circ *$ & $\circ *$ & $\circ *$ & $\circ *$ & $\circ *$ & $\circ *$ & ०* \\
\hline 708 & $\circ *$ & $\circ *$ & $\circ *$ & $*$ & * & $*$ & $*$ & 0 & $\circ *$ & & & \\
\hline 700 & $\circ *$ & $*$ & $*$ & & & $\circ *$ & $\circ *$ & $\circ *$ & $\circ *$ & $\circ$ & $\circ$ & o* \\
\hline 701 & $*$ & $*$ & $*$ & $\circ *$ & * & & $\circ *$ & $*$ & $\circ *$ & $\circ *$ & * & o \\
\hline 702 & o* & $\circ *$ & $\circ *$ & $*$ & $*$ & $\circ *$ & $\circ *$ & $\circ *$ & $\circ$ & $\circ *$ & $\circ$ & $\circ *$ \\
\hline 607 & & $\circ *$ & $\circ *$ & & & & & $\circ *$ & & $\circ$ & & \\
\hline 595 & $*$ & $*$ & $*$ & & & & $\circ$ & $*$ & $\circ *$ & $*$ & $*$ & \\
\hline 596 & o* & $\circ *$ & $\circ *$ & $\circ *$ & $\circ *$ & $\circ *$ & $\circ *$ & $*$ & $\circ *$ & ○ & * & * \\
\hline 602 & & * & $\circ *$ & $\circ$ & & $\circ$ & & $\circ *$ & $*$ & $\circ$ & $\circ$ & \\
\hline 712 & $\circ *$ & $\circ *$ & $\circ *$ & $*$ & $\circ *$ & $\circ *$ & $\circ *$ & $*$ & $\circ$ & $\circ *$ & $\circ$ & $\circ *$ \\
\hline 714 & $\circ *$ & $\circ *$ & $\circ *$ & $\circ *$ & $\circ *$ & $\circ *$ & $\circ *$ & o* & $\circ *$ & $\circ *$ & ० & o* \\
\hline
\end{tabular}

Fonte: Autores.

Para a correlação de Spearmam (Tabela 2) considerou-se a espécie em geral e o agrupamento dos 66 acessos em cinco grupos (Estados) de acordo com a procedência do material. De maneira geral, a ipeca apresentou para a variável frutificação correlação significativa forte (rho forte $>0,67$ ) inversamente proporcional para a temperatura (rho $=-0.7605822, \mathrm{P}$ $=0.004078)$ e diretamente proporcional para a precipitação ( $r h o=0.6853147 * \mathrm{P}=0.01731)$, o que permite inferir que quanto menor a temperatura e maior a precipitação, maior será a incidência de frutos (Tabela 2, Figura 4A e B). Este estudo esta de acordo com Santana et al, (2020) em que nota correlação negativa $(-0,98)$ da temperatura do ar com o número de dias decorrido para a frutificação. Isto é, quanto maior a temperatura do ar, menor será o número de dias para frutificação. Segundo Chagas et al., (2019), a atividade dos frutos maduros de Elaeis guineensis teve correlação significativa e positiva também com a umidade e a precipitação. Portanto, quanto maior a umidade e quanto maior o volume pluviométrico, maior é a produção de frutos maduros.

Com relação à floração da espécie e as variáveis climáticas notasse que ocorreu correlação não significativa e negativa tanto para a temperatrura (rho $=-0.33333$ e P = 0.2897) quanto para a precipitação(rho $=-0.33333$ e P 0.2897). Houve também correlações não significativas ou significativas, fortes ou fracas entre os acessos, quando se observa os distintos grupos. Para a variável floração quatro grupos não se correlacionaram com os fatores bióticos apenas os acessos de Mato Grossos se correlacionaram de forma significativa e positiva com a temperatura. Quanto à frutificação os grupos de acessos de Rondônia, Rio de Janeiro se correlacionaram significativamente com os fatores climáticos e o grupo de acessos de Mato Grosso teve correlação significativa apenas com a temperatura, para os demais a correlação não foi significativa nesta fenofase (Tabela 2). 
Tabela 2. Correlações de Spearman (rho) entre a média mensal das variáveis climáticas e os eventos fenológicos de acessos de Ipeca provenientes de cinco Estados,BR.

\begin{tabular}{llllll}
\hline \multirow{2}{*}{ Procedência } & \multirow{2}{*}{ Fatores abióticos } & \multicolumn{2}{c}{ Floração } & \multicolumn{2}{c}{ Frutificação } \\
\cline { 3 - 6 } & & rho & $\mathrm{p}$ & rho & $\mathrm{P}$ \\
\hline Rondônia & Temperatura & $0.206407 \mathrm{~ns}$ & 0.5198 & $-0.8663225^{* 2}$ & 0.0002675 \\
\multirow{2}{*}{ Minas Gerais } & Precipitação & $-0.04240 \mathrm{~ns}$ & 0.8959 & $0.7080189^{*}$ & 0.00998 \\
& Temperatura & $-0.06523 \mathrm{~ns}$ & 0.8404 & $-0.5895063^{* 1}$ & 0.04367 \\
Bahia & Precipitação & $0.129550 \mathrm{~ns}$ & 0.6882 & $0.529619 \mathrm{~ns}$ & 0.07657 \\
\multirow{2}{*}{ Rio de janeiro } & Temperatura & $0.028071 \mathrm{~ns}$ & 0.931 & $-0.48377 \mathrm{~ns}$ & 0.111 \\
& Precipitação & $0.139373 \mathrm{~ns}$ & 0.6657 & $0.305699 \mathrm{~ns}$ & 0.3339 \\
Mato Grosso & Temperatura & $0.01684 \mathrm{~ns}$ & 0.9586 & $-0.7914452^{* 2}$ & 0.002163 \\
& Precipitação & $0.192335 \mathrm{~ns}$ & 0.5493 & $0.7897816 * 2$ & 0.002244 \\
Espécie & Temperatura & $0.61947 * 1$ & 0.0317 & $-0.40212 \mathrm{~ns}$ & 0.195 \\
& Precipitação & $-0.48155 \mathrm{~ns}$ & 0.1129 & $0.479860 \mathrm{~ns}$ & 0.1144 \\
& Temperatura & $-0.33333 \mathrm{~ns}$ & 0.2897 & $-0.7605822^{* 2}$ & 0.004078 \\
& Precipitação & $-0.33333 \mathrm{~ns}$ & 0.2897 & $0.6853147 * 2$ & 0.01731 \\
\hline
\end{tabular}

rho $=$ coeficiente de correlação de Spearman

$* \mathrm{P}<0,05=$ Valores significativos

Rho forte $>0,67=\left({ }^{2}\right)$; rho fraca entre $<0,66=\left({ }^{1}\right)$

Fonte: Autores.

Embora a correlação de Spearman considerando a espécie como um todo a floração não ter se correlacionado significativamente com as variáveis climáticas, a Figura 3 permite observar variações na ocorrência de número de dias de floração e frutificação entre os grupos de acessos. O registro fenológico mostrou as máximas concentrações de grupos de acessos que apresentaram maiores médias de percentual de dias de ocorrência de floração em outubro com 24\% (MG) e em novembro com 16\% (RO), e com valores médios de 149,2 e 134,5 mm de precipitação pluviométrica, período de estiagem (Figura 3A), e temperatura média do ar de 27,9 e $28^{\circ} \mathrm{C}$, respectivamente, sendo estas as maiores médias desta variável (Figura 3B).Houve uma redução na frequência de dias desta fenofase nos grupos de acessos avaliados de abril á agosto, coincidindo com períodos em que a temperatura média começa a elevar, na transição do período chuvoso ao verão amazônico (Figura 3B). Resultados semelhantes foram obtidos por Rocha (2011), onde os maiores dias de floração, coincidindo com os meses de menor precipitação pluviométrica. Para Nascimento et al, (2020) em estudo acerca da fenologia reprodutiva das espécies vegetais relacionadas aos fatores climáticos, embora não tenha apresentado correlação estatística significativa, grande parte delas demonstrou maiores padrões de intensidade. 
Figura 3. Percentual de dias por mês de ocorrência do evento fenológico floração da Ipeca, no período de janeiro de 2015 a dezembro de 2019.
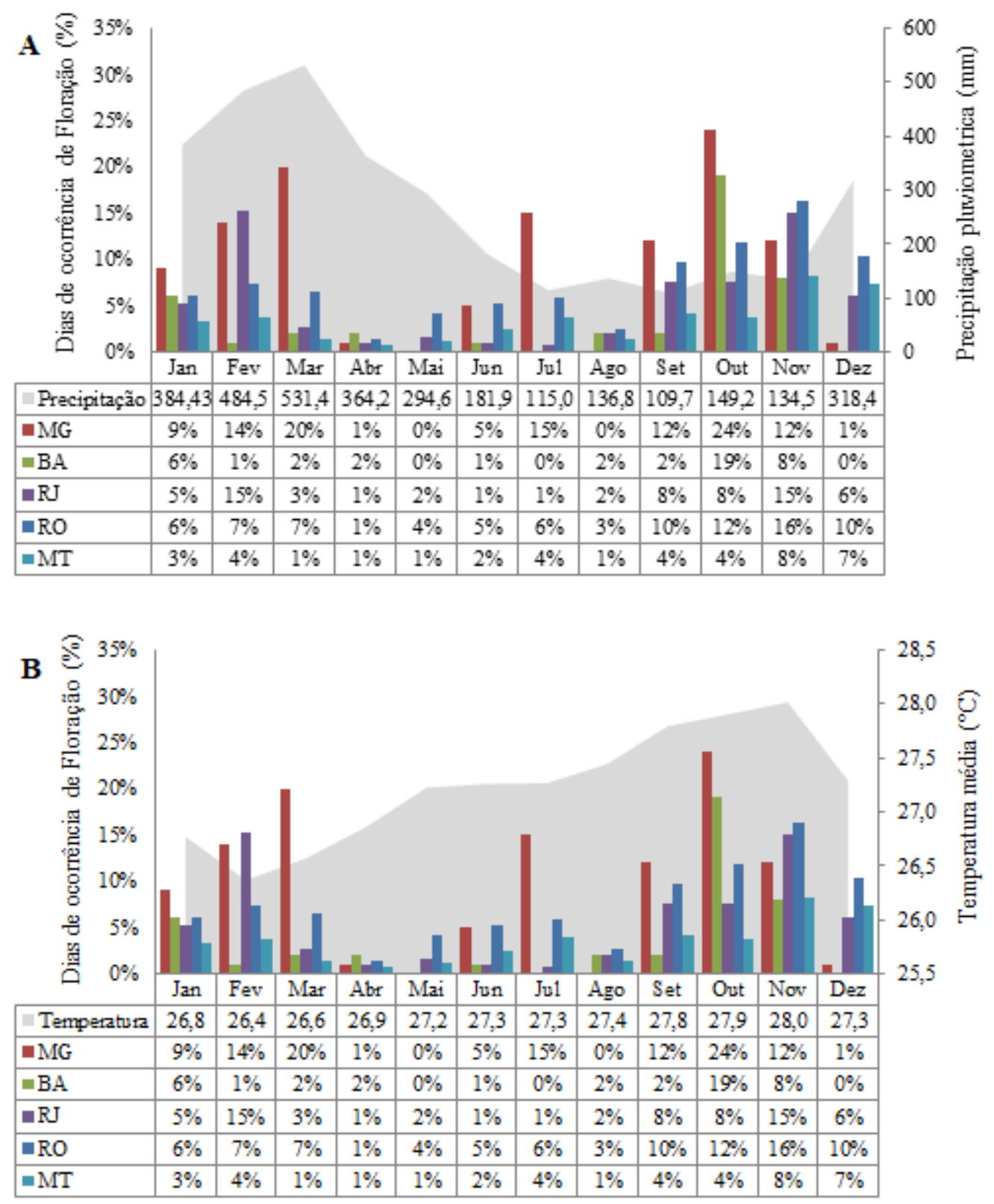

Fonte: Autores.

O evento fenológico de frutificação registrou as maiores médias percentuais de dias de ocorrência para os meses de fevereiro á junho, sendo o maior número registrado simultaneamente em março e abril (20\%, BA), coincidindo com o valor de alto índice de precipitação pluviométrica (531,4 e 364,2 mm), que se enquadram no período do inverno amazônico (Figura 4. A), em relação às temperaturas médias para os meses de maior ocorrência de dias de frutificação foram 26,6 e $26,9^{\circ} \mathrm{C}$, respectivamente. Uma redução na frequência de dias desta fenofas e nos grupos de acessos avaliados foi registrada de agosto á novembro, coincidindo com períodos em que a temperatura começa a elevar (Figura 4B). A temperatura é influente no que diz respeito às alterações fenológicas (formação de frutos) das espécies vegetais em um determinado local (Hidalgo-Gavezet al., 2018). Neto. (2020) evidenciou que a fenologia da espécie frutifera Psidium cattleianum responde diretamente à oscilação de temperatura e precipitação. 
Figura 4. Percentual de dias por mês de ocorrência do evento fenológico frutificação de Ipeca, no período de janeiro de 2015 a dezembro de 2019.

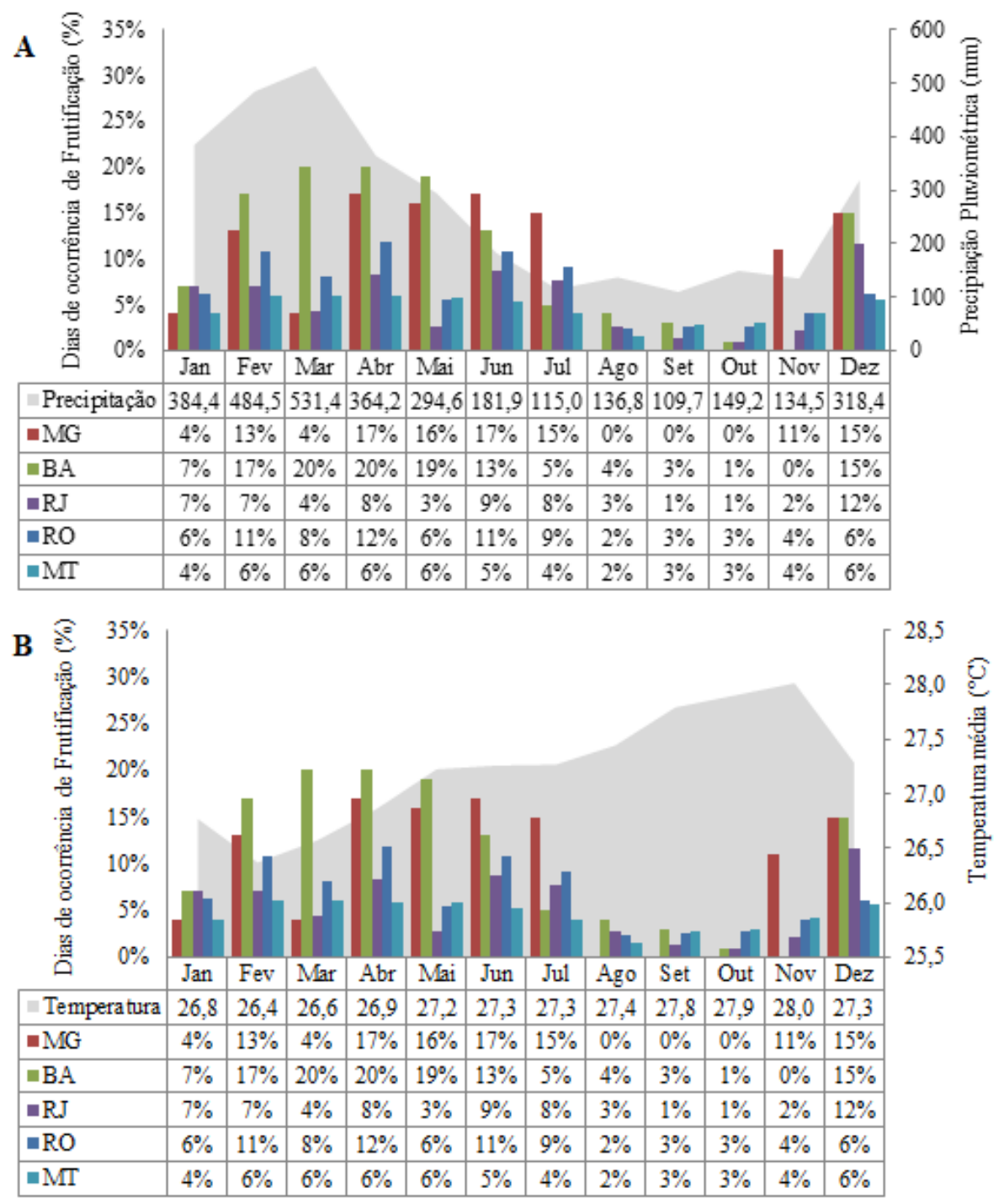

Fonte: Autores.

\section{Conclusão}

O estudo fenológico da espécie Carapichea ipecacuanha evidenciou variações no padrão dos eventos fenológicos reprodutivos, atividade fenológica dissimilares entre acessos, além de correlações entre frutificação e variáveis climáticas com significância, intensidade e proporções desiguais entre os grupos de acessos observados. A fase de frutificação da espécie esta correlacionada fortemente de forma negativa com a temperatura e positiva com a precipitação pluviométrica. Contudo sugerese a coleta de material vegetal da espécie para uso medicinal nos meses de agosto e setembro, cujo registro de número de dias que ocorrem as fenofases sofre reduções. È pertinente executar trabalhos posteriores que abranjam o estudo de correlação da fenologia com eventos climáticos avaliando o desempenho individual de cada acesso.

\section{Agradecimentos}

Os autores agradecem à Coordenação de Aperfeiçoamento de Pessoal de Nível Superior (CAPES) pelo apoio e bolsa de estudo de Doutorado, a Embrapa Amazônia Oriental por ceder o local e a estrutura e ao Programa de Doutorado da Rede 
Bionorte - Biodiversidade e Biotecnologia.

\section{Referências}

Bencke, C. S., \& Morellato, L. P. C. (2002). Comparação de dois métodos de avaliação da fenologia de plantas, sua interpretação e representação. Brazilian Journal of Botany, 25(3), 269-275.

Embrapa. (2021). Embrapa Recursos Genéticos E Biotecnologia. Núcleo de Tecnologia da Informação (NTI). AleloVegetal.

Ferreira, Q. S., Paloma, M., Lima dos Santos, L., \& Trindade, M. F. (2012). Use and importance of quina (Cinchona spp.) and ipeca (Carapichea ipecacuanha (Brot.) L. Anderson): Plants for medicinal use from the 16th century to the present. J Herb Med, 2(4), 103-112.

Forsthofer, E. L., Silva, P. R. F. D., Argenta, G., Strieder, M. L., Suhre, E., \& Rambo, L. (2004). Desenvolvimento fenológico e agronômico de três híbridos de milho em três épocas de semeadura. Ciência Rural, 34(5), 1341-1348.

Fortunato, M. E. M., \& Quirino, Z. G. M. (2016). Efeitos da fragmentação na fenologia reprodutiva de espécies arbóreas presentes em borda e interior de Mata Atlântica Paraibana. Rodriguésia, 67(3), 603-614.

Forzza, R. C.; Costa, A.; Walter, B. M .T., Pirani, J. R., Morim, M. P., Queiroz, L. P., Martinelli, G., Peixoto, A. L., Coelho, M. A. N., Baumgratz, J. F. A., Stehmann, J. R., \& Lohmann, L. G. Angiospermas in: Lista De Espécies Da Flora Do Brasil. Jardim Botânico do Rio de Janeiro. 2014. http://reflora.jbrj.gov.br/jabot/floradobrasil/FB128482.

Hidalgo-Galvez, M. D., García-Mozo, H., Oteros, J., Mestre, A., Botey, R., \& Galán, C. (2018). Phenological behaviour of early spring flowering trees in Spain in response to recent climate changes. Theoretical and applied climatology, 132(1), 263-273.

Kosera Neto, C., Radaelli, J. C., Guollo, K., Wagner Júnior, A., Zanela, J., \& Frazon, R. C. (2020). Acompanhamento fenológico de araçazeiros na Região Sudoeste do Paraná. Research, Society and Development, 9(10), e8319109180. https://doi.org/10.33448/rsd-v9i10.9180.

Lopes Ribeiro, C., Ferreira de Melo Jr, J. C., Dubet da Silva Mouga, D. M., \& Carneiro, E. (2018). Fenologia e visitantes florais de Psychotria nuda (Rubiaceae) em um fragmento urbano de Mata Atlântica no sul do Brasil. Neotropical Biology \& Conservation, 13(3).

Lopes, L. E.,\& Buzato, S. (2005). Biologia reprodutiva de Psychotria suterella Muell. Arg.(Rubiaceae) e a abordagem de escalas ecológicas para a fenologia de floração e frutificação. Brazilian Journal of Botany, 28(4), 785-795.

Morellato, L. P. C., Alberton, B., Alvarado, S. T., Borges, B., Buisson, E., Camargo, M. G. G., \& Peres, C. A. (2016). Linking plant phenology to conservation biology. Biological Conservation, 195, 60-72.

Nascimento, G. M., dos Santos Oliveira, F., \& do Nascimento, I. O. (2020). Dados fenológicos de espécies vegetais da reserva extrativista ciriaco em cidelândia e imperatriz, maranhão. Revista Eletrônica Científica Ensino Interdisciplinar, 6(19).

Neves, E. L. D., Funch, L. S., \& Viana, B. F. (2010). Comportamento fenológico de três espécies de Jatropha (Euphorbi aceae) da Caatinga, semi-árido do Brasil. Brazilian Journal of Botany, 33(1), 155-166.

Pascale,A. J., \& Damario, E. A. Biocimatolgia Agrícola y Agroclimatolocía. Buenos Aires: Editorial Facultad Agronomia, Universidad de Buenos Aires, 2004, $550 \mathrm{p}$.

Peel, M. C., Finlayson, B. L., \& McMahon, T. A. (2007). Updated world map of the Köppen-Geiger climate classification. Hydrology and earth system sciences, $11(5), 1633-1644$.

R Core Team (2020). R: A languageandenvironment for statisticalcomputing. R Foundation for StatisticalComputing, Vienna, Austria. https://www.Rproject.org/.

Ricardo, L. M., de Paula-Souza, J., Andrade, A., \& Brandão, M. G. (2017). Plants from the Brazilian traditional medicine: species from the books of the Polish physician Piotr Czerniewicz (Pedro Luiz Napoleão Chernoviz, 1812-1881). Revista Brasileira de Farmacognosia, 27(3), 388-400.

Rocha, T. T., \& Lameira, O. A. (2011). Avaliação do período de floração e frutificação do BAG Ipecacuanha. In Embrapa Amazônia Oriental-Artigo em anais de congresso (ALICE). In: Seminário De Iniciação Científica Da Embrapa Amazônia Oriental, 15.,2011, Belém, PA. A ciência de fazer ciência: anais. Belém, PA: Embrapa Amazônia Oriental.

Santana, A. S., Giacobbo, C. L., do Prado, J., Uberti, A., Louis, B., \& Alberto, C. M. (2020). Fenologia e qualidade de frutos de acessos de Physalis spp. Agrarian, 13(47), 1-8.

Silva, M. L. D. (2014). Cultivo agroflorestal de Psychotria Ipecacuanha (Brot.) Stokes no território do baixo sul da Bahia. 4.75 f. Dissertação (Mestrado em Recursos Genéticos Vegetais) - Universidade Estadual de Feira de Santana, Feira de Santana.

Tannus, J. L., Assis, M. A., \& Morellato, L. P. C. (2006). Fenologia reprodutiva em campo sujo e campo úmido numa área de cerrado no sudeste do Brasil, Itirapina-SP. Biota Neotropica, 6(3), 0-0.

Tropicos. Tropicos.org. Missouri Botanical Garden. 2014. http://www.tropicos.org .

Vieira, F. D. A., \& Carvalho, D. D. (2009). Maturação e morfometria dos frutos de Miconia albicans (Swartz) Triana (Melastomataceae) em um remanescente de floresta estacional semidecídua montana em Lavras, MG. Revista Árvore, 33(6), 1015-1023.

Zanon, M. L. B., \&Finger, C. A. G. (2010). Relação de variáveis meteorológicas com o crescimento das árvores de Araucaria angustifolia (Bertol.) Kuntze em povoamentos implantados. Ciência Florestal, 20(3), 467-476. 